\title{
Impact of Vitamin C Supplementation on Post-Cardiac Surgery ICU and Hospital Length of Stay
}

\author{
Anita Sadeghpour ${ }^{1}$; Azin Alizadehasl ${ }^{1}$; Majid Kyavar ${ }^{1,{ }^{*}}$; Tahereh Sadeghi ${ }^{1}$; Jalal Moludi ${ }^{2}$; \\ Farhad Gholizadeh ${ }^{2}$; Ziae Totonchi ${ }^{1}$; Behshid Ghadrdoost ${ }^{1}$ \\ ${ }^{1}$ Rajaie Cardiovascular Medical and Research Center, Iran University of Medical Sciences, Tehran, Iran \\ ${ }^{2}$ School of Nutritional Sciences and Dietetics, Tehran University of Medical Sciences, Tehran, Iran \\ ${ }^{*}$ Corresponding author: Majid Kyavar, Rajaie Cardiovascular Medical and Research Center, Iran University of Medical Sciences, Tehran, Iran. Tel: +98-2123922198, E-mail: kiavar@rhc. \\ ac.ir
}

Received: November 14, 2014; Revised: December 11, 2014; Accepted: December 15, 2014

\begin{abstract}
Background: Prolonged intensive care unit (ICU) and hospital stay after cardiac surgery is vitally important and is influenced by both intraoperative and postoperative factors.

Objectives: This randomized clinical trial study was designed to assess whether vitamin C supplementation could reduce the length of ICU and hospital stay in post-cardiac surgery patients.

Patients and Methods: Two hundred and ninety patients scheduled for adult cardiac surgery including coronary, valve and congenital operations were randomized into two groups: an intervention group, who received $2 \mathrm{~g}$ of vitamin C (ascorbic acid) intravenously, immediately before surgery in the operating theatre, followed by $1 \mathrm{~g}$ daily oral doses for the first 4 postoperative days; and a placebo group, who received an equal number of identical tablets in the same shape and size.

Results: Hospital length of stay was significantly different between the two groups (10.17 \pm 4.63 days in the intervention group vs. $12 \pm$ 4.51 days in the placebo group; $\mathrm{P}=0.01$ ), while there was no significant difference in the ICU stay between the groups (3.42 \pm 1.06 days in intervention group vs. $3.43 \pm 1.09$ days in the placebo group; $\mathrm{P}=0.88$ ). There were significant differences in the intubation time and the drainage volume in the ICU and the first 24 postoperative hours between the two groups (P for both $=0.003$ ).

Conclusions: Vitamin C can decrease the length of hospital stay, drainage volume in the ICU and in the first 24 postoperative hours, intubation time and some complications in patients after cardiac surgery; perhaps by decreasing inflammatory factors.
\end{abstract}

Keywords:Vitamin C; Antioxidant; Intensive Care Unit; Hospital Stay; Atrial Fibrillation

\section{Background}

The length of stay in the intensive care unit(ICU) is an important issue in contemporary cardiac surgery and in the management of health care resources around the globe, not least given the rise in the ageing population and longevity of high-risk patients $(1,2)$. Several factors can affect the length of stay in the ICU and hospital such as disease severity, bypass or cross-clamp time, endotracheal intubation time, hemodynamic instability, older age and comorbidities. Moreover, postoperative bleeding, need for reoperation and postoperative arrhythmias are among the factors having been associated with an increased length of stay in the ICU, followed by a longer hospital stay (3).

Recent years have witnessed great strides taken to reduce complications and improve the outcome of patients by shortening the ICU or hospital length of stay. To that end, therapeutic approaches, especially administration of antioxidants, have been accorded distinctive attention. Many studies have shown that vitamin C, deemed the most important antioxidant, may attenuate electrical remodeling, decrease the incidence of postop- erative atrial fibrillation (AF) (4), alleviate postoperative pain and reduce the incidence of pulmonary morbidity and dysfunction of the other organs (5-8). Postulating that vitamin C supplementation in cardiac surgery patients might reduce the incidence of complications and ICU and hospital length of stay, we conducted the present randomized clinical trial to evaluate the effect of this antioxidant on the length of ICU and hospital stay, drainage volume, need for reoperation and some related factors after cardiac surgery.

\section{Objectives}

This randomized clinical trial study was designed to assess whether vitamin $C$ supplementation could reduce the length of ICU and hospital stay in post-cardiac surgery patients.

\section{Patients and Methods}

This randomized, double-blind, placebo-controlled

Copyright (C) 2015, Iranian Society of Regional Anesthesia and Pain Medicine(ISRAPM). This is an open-access article distributed under the terms of the Creative Commons Attribution-NonCommercial 4.0 International License (http://creativecommons.org/licenses/by-nc/4.0/) which permits copy and redistribute the material just in noncommercial usages, provided the original work is properly cited. 
prospective trial was conducted in patients undergoing cardiac surgery in Rajaie Cardiovascular, Medical and Research Center, Tehran, Iran. The study protocol was in accordance with the Helsinki Declaration of the World Medical Association (2000) and was approved by our local ethics committee. Written informed consent was obtained from all the patients. The inclusion criteria comprised age $\geq 18$ years with American Society of Anesthesiologists (ASA) physical status class II-III and candidacy for coronary artery bypass graft operation (CABG) or simple congenital valvular disease surgery. Patients who died within the first postoperative day and those who had not received adequate doses of drugs according to our protocol were excluded. Also, we excluded the patients were severe complications (cardiac, respiratory or neurological) or emergent operation.

\subsection{Randomisation and Interventions}

The study population was randomized one day before surgery to two groups (By using www.randomaizer.org): the intervention (vitamin $\mathrm{C}$ supplementation) group and the placebo group. The method of randomization was balanced block with an allocation sequence based on a block size of eight, generated with a computer randomnumber generator. Both the patients and the hospital staff were blind to the treatment allocation. The patients in the intervention group received $2 \mathrm{~g}$ of vitamin $\mathrm{C}$ (ascorbic acid; Darou Pakhsh Co., Tehran, Iran) intravenously, immediately before surgery in the operating theatre, followed by $1 \mathrm{~g}$ daily oral doses of the tablets for the first four postoperative days. The patients in the placebo group received an equal number of identical tablets. The placebo tablets and ampoules were prepared in the same shape and size as the original ones manufactured by the same pharmaceutical company. Both groups had the same surgical premedication and anesthesia protocol as well as surgical access (median sternotomy).

Before surgery, basic information such as age, sex and risk factors was collected, and medications were given to all the patients according to their clinical indications. All the patients were monitored in the open heart ICU before their transfer to a ward. Data such as bypass time and cross-clamp time were collected intraoperatively. Additionally, information was gathered in the ICU on the total length of ICU stay, intubation time, drainage volume and presence of some complications (mortality and morbidity) defined as death, any infection, any impairment in the renal function and need for reoperation. Finally, the total duration of hospital stay was recorded for every individual.

\subsection{Statistical Analysis}

The statistical analyses were performed with SPSS 15 for Windows (SPSS Inc., Chicago, Illinois). P values $\leq$ 0.05 were considered significant. The data are expressed as mean values \pm standard deviation (SD) for the inter- val and count (\%) for the categorical variables. All the variables were tested for normal distribution with the Kolmogorov-Smirnov test. The categorical values were compared using the chi-square test or the Fisher exact test. The mean variables were compared between the two groups using an independent t-test or the MannWhitney U test.

A binary logistic regression analysis was performed to adjust the confounding factors of ICU and hospital length of stay. These variables included those that had an association of $\mathrm{P}<0.05$ with hospital and ICU length of stay in the univariate model.

\section{Results}

The study population consisted of 290 patients at a mean age of $55.78 \pm 13.72$ years (18-85 years). The intervention group comprised 113 patients, and the placebo group included 177 patients. The baseline and surgery-related characteristics of the vitamin $\mathrm{C}$ supplementation and placebo groups are depicted in Table 1 . There were no significant differences between the groups regarding the measured baseline and perioperative characteristics (age, sex, risk factors and surgery type).

The intraoperative variables were also compared between the two groups (Table 1 ). No difference was found in surgery type and on/off-pump CABG between the groups. There was a significant difference in terms of the intubation time between the two groups $(11.83 \pm 3.91 \mathrm{vs} .14 .14 \pm$ 9.52 hours; $\mathrm{P}=0.003)$, whereas the differences between the study groups regarding bypass time and cross-clamp time constituted no statistically significant differences. The drainage volume in the ICU and in the first 24 postoperative hours differed significantly between the intervention and placebo groups $(\mathrm{P}=0.003)$. Also importantly, a significant difference existed between the two groups of patients as regards postoperative complications, defined as death, any infection and any impairment in the renal function $(\mathrm{P}=0.042)$. Although the rate of need for reoperation was higher in the intervention group, the difference was not statistically significant between the two groups $(P=0.3)$. Hospital length of stay was significantly different between the two groups, but the difference in ICU length of stay between the studied groups failed to reach statistical significance. The mean ICU length of stay after surgery was $3.42 \pm 1.06$ days in the vitamin $C$ supplementation group and $3.43 \pm 1.09$ days in the placebo group $(\mathrm{P}=0.88)$. The mean hospital length of stay was $10.17 \pm 4.63$ days among the patients in the intervention group and $12 \pm 4.51$ days among those in the placebo group $(\mathrm{P}=0.01)$ (Table 2$)$.

The overall incidence rate of postoperative AF in the vitamin C supplementation group was 40 (35.5\%), versus 99 (55.9\%) in the placebo group ( $\mathrm{P}=0.001)$. The logistic regression analysis showed that AF occurrence was not a confounding factor in the length of hospital or ICU stay (Table 3). 
Sadeghpour A et al.

Table 1. Perioperative, Intraoperative and Postoperative Characteristics of the Patients ${ }^{a, b}$

\begin{tabular}{|c|c|c|c|}
\hline Variables & Vitamin $C(n=113)$ & $\operatorname{Placebo}(\mathbf{n}=177)$ & P Value \\
\hline \multicolumn{4}{|l|}{ Gender } \\
\hline Male & $80(70.8)$ & $111(62.7)$ & 0.15 \\
\hline Female & $33(29.2)$ & $66(37.3)$ & 0.15 \\
\hline Age, y & $57.28 \pm 14.09$ & $54.22 \pm 14.39$ & 0.70 \\
\hline HT & $52(49.5)$ & $73(46.5)$ & 0.63 \\
\hline DM & $27(26.2)$ & $37(24)$ & 0.69 \\
\hline $\mathbf{C} / \mathbf{S}$ & $18(17.6)$ & $26(16.7)$ & 0.83 \\
\hline HLP & $10(38.5)$ & $22(34.4)$ & 0.71 \\
\hline \multicolumn{4}{|l|}{ Surgery type } \\
\hline CABG & $57(50.4)$ & $94(53.1)$ & 0.44 \\
\hline Valvular & $30(26.5)$ & $52(29)$ & 0.44 \\
\hline Mixed & $20(17.6)$ & $22(12.4)$ & 0.44 \\
\hline Congenital & $6(5.3)$ & $9(5.5)$ & 0.44 \\
\hline \multicolumn{4}{|l|}{ Operation } \\
\hline Off-pump & $12(11.2)$ & $16(9.4)$ & 0.62 \\
\hline On-pump & $95(88.8)$ & $154(90.6)$ & 0.62 \\
\hline Reoperation & $7(6.2)$ & $17(9.6)$ & 0.30 \\
\hline Complications $^{\mathrm{c}}$ & $4(3.54)$ & $18(10.2)$ & 0.042 \\
\hline Bypass time, min & $97.17 \pm 42.62$ & $86.98 \pm 37.98$ & 0.06 \\
\hline Cross-clamp time, min & $55.93 \pm 30.32$ & $50.89 \pm 30.84$ & 0.08 \\
\hline Intubation time, $h$ & $11.83 \pm 3.91$ & $14.14 \pm 9.52$ & 0.003 \\
\hline Drainage in the first 24 postoperative hours (cc) & $262.21 \pm 190.91$ & $348.50 \pm 262.17$ & 0.003 \\
\hline Drainage in ICU (cc) & $499.55 \pm 296.69$ & $577.96 \pm 454.44$ & 0.107 \\
\hline
\end{tabular}

a Abbreviations: HT, Hypertension; DM, Diabetes Mellitus; C/S, Cigarette smoking; HLP, Hyperlipidaemia; CABG, Coronary Artery Bypass Grafting.

${ }^{b}$ The data are expressed as mean \pm SD for the interval and No. (\%) for the categorical variables.

${ }^{\mathrm{c}}$ Some Complications Including: Death, Impaired Renal Function and Infection.

Table 2. ICU and Hospital Length of Stay Comparison Between the Two Groups a,b

\begin{tabular}{lccc}
\hline Variables & Vitamin $\mathbf{C}(\mathbf{n}=\mathbf{1 1 3})$ & Placebo $(\mathbf{n}=\mathbf{1 7 7})$ & PValue \\
\hline ICU Stay & $3.42 \pm 1.06$ & $3.43 \pm 1.09$ & 0.88 \\
Hospital Stay & $10.17 \pm 4.63$ & $12 \pm 4.51$ & 0.01 \\
\hline
\end{tabular}

a Abbreviation: ICU, Intensive Care Unit.

$\mathrm{b}$ The data are expressed as mean $\pm \mathrm{SD}$.

Table 3. Logistic Regression for Adjusting AF as a Confounding Factor ${ }^{a}$

\begin{tabular}{lcccc}
\hline Variables & B & Beta & t & PValue \\
\hline Hospital Stay & & & & \\
$\quad$ Intervention & 0.84 & 0.09 & 1.50 & 0.13 \\
AF & -0.68 & -0.74 & -1.24 & 0.21 \\
ICU Stay & & & 0.09 & 0.92 \\
$\quad$ Intervention & 0.01 & 0.006 & -0.14 & 0.88 \\
\hline AF & -0.01 & -0.009 & & \\
\hline
\end{tabular}

a Abbreviations: AF, Atrial Fibrillation; ICU, Intensive Care Unit. 
Sadeghpour A et al.

\begin{tabular}{lcccc}
\hline \multicolumn{1}{l}{ Table 4. Binary Logistic Regression Analysis of Factors Affecting the Length of ICU Stay ${ }^{\text {a }}$} & & \multicolumn{1}{l}{} \\
\hline Variables & B & Beta & t & P Value \\
\hline Intervention & 0.011 & 0.005 & 0.077 & 0.93 \\
Bypass Time & 0.003 & 0.095 & 0.727 & 0.46 \\
Cross-Clamp Time & 0.002 & 0.044 & 0.345 & 0.73 \\
Intubation Time & 0.017 & 0.135 & 2.085 & 0.03 \\
Drainage in the First 24 & 0.000 & -0.061 & -0.444 & 0.65 \\
Postoperative Hours (cc) & & & 2.439 & 0.01 \\
Drainage in ICU(cc) & 0.001 & 0.329 & -0.239 & 0.81 \\
Drainage Time & 0.000 & -0.015 & 0.973 & 0.33 \\
Kind of Surgery & 0.105 & 0.072 & 0.050 & 0.96 \\
Reoperation & 0.013 & 0.003 & 2.303 & 0.02 \\
\hline Complications & 0.561 & 0.152 & & \\
\hline
\end{tabular}

a Abbreviation: ICU, Intensive Care Unit.

\begin{tabular}{|c|c|c|c|c|}
\hline Variables & B & Beta & $\mathbf{t}$ & PValue \\
\hline Intervention & 0.660 & 0.067 & 1.069 & 0.286 \\
\hline Drainage in ICU (cc) & 0.000 & 0.049 & 0.738 & 0.461 \\
\hline Drainage Time & -0.011 & -0.084 & -1.364 & 0.174 \\
\hline Intubation Time & 0.084 & 0.150 & 2.327 & 0.021 \\
\hline Kind of Surgery & 1.148 & 0.181 & 2.649 & 0.009 \\
\hline Reoperation & -2.155 & -0.133 & -1.929 & 0.055 \\
\hline Operation & 0.457 & 0.028 & 0.441 & 0.659 \\
\hline Complications & 1.524 & 0.092 & 1.444 & 0.150 \\
\hline
\end{tabular}

a Abbreviation: ICU, Intensive Care Unit.

Based on the univariate analysis, the intubation time, bypass time, drainage volume in the ICU and in the first 24-hour postoperative period, drainage time, surgery type, need for reoperation and complications had significant relationships with the length of stay in the ICU. The logistic regression model showed that the intubation time (odds ratio [OR]: $2.08, \mathrm{P}=0.03$ ) and presence of complications (OR: 2.30, $\mathrm{P}=0.02$ ) were the independent factors of the ICU length of stay (Table 4).

The univariate analysis demonstrated that the drainage volume in the ICU and in the first 24 postoperative hours, drainage time, kind of surgery, on/off-pump CABG, need for reoperation and presence of complications had significant relationships with the length of hospital stay. The logistic regression model showed that the intubation time (OR: 2.32, $\mathrm{P}=0.02$ ) and surgery type (OR: 2.64, $\mathrm{P}$ $=0.009$ ) were the independent factors of hospital length of stay (Table 5).

\section{Discussion}

A significant body of evidence suggests that antioxidant vitamins, especially vitamin $\mathrm{C}$, have an important role in preventing a number of complications after certain op- erations, including cardiac surgery (4, 8-11). Many studies have demonstrated a strong relationship between the usage of vitamin $C$ and a reduction in the incidence of postoperative $\operatorname{AF}(5,10,11)$. Furthermore, the reduce in development of pulmonary morbidity and organ failure in the early administration of ascorbic acid supplementation may be due to a decrease in inflammatory and oxidative factors (5). The present study was a randomized placebocontrolled trial in which antioxidant vitamin $C$ was given to patients who underwent cardiac surgery to assess the length of ICU and hospital stay as well as some related factors. The two study groups, intervention and placebo, were similar preoperatively: there were no differences between the vitamin $C$ supplementation group and the placebo group in terms of demographic characteristics, cardiovascular risk factors such as age, sex, diabetes mellitus, hypertension, hyperlipidaemia and cigarette smoking and also surgery kind.

In the current study, in accordance with previous studies regarding the effect of vitamin $C$ supplementation on patient outcomes after cardiac surgery, we showed that vitamin $\mathrm{C}$ led to a decrease in mortality in the ICU, length of hospital stay, postoperative drainage volume, intubation time and some important complications. 
There was, however, no statistically significant effect on the length of stay in the ICU. Nathens et al. in their randomized trial study demonstrated that the early administration of ascorbic acid supplementation shortened ICU length of stay by reducing the duration of mechanical ventilation (5), which chimes in with our findings insofar as the intubation time in the vitamin $C$ group was significantly lower than that in the placebo group (11.83 \pm 3.91 hours in the vitamin C group vs. $14.14 \pm 9.52$ hours in the placebo group; $\mathrm{P}=0.003$ ). Our univariate analysis showed that some of the peri- and postoperative characteristics of the patients were associated with the length of both ICU and hospital stay. The patients who underwent on-pump CABG had a longer ICU and hospital stay than those who underwent off-pump surgery. The length of both ICU and hospital stay increased in the patients undergoing mixed surgery (i.e. simultaneous CABG and valvular surgery) and in those needing reoperation. Furthermore, we observed a trend towards a longer length of ICU and hospital stay in the patients with prolonged bypass time and cross-clamp time in the operating theatre. Our results revealed that the patients' postoperative characteristics exerted a more significant effect on the length of ICU and hospital stay inasmuch as the duration of endotracheal intubation and the volume of drainage in the ICU and in the first 24 postoperative hours were significantly associated with a longer stay in the ICU or hospital.

Our binary logistic regression analysis of the factors affecting the length of both ICU and hospital stay illustrated that the most important factor in increasing the length of hospital and ICU stay was the intubation time. In addition, a shorter length of ICU and hospital stay was achieved when the cardiac surgical patients were weaned from the mechanical ventilator at the appropriate time. There is some evidence showing that a prolonged intubation time is associated with a greater frequency of complications and prolongation of the ICU stay (12-14). Since our results showed that the intubation time was shorter in the patients who received vitamin $\mathrm{C}$, it can be concluded that vitamin $C$, by extension, can affect the length of stay in hospital. We examined the incidence of AF as an important factor, which can increase the length of hospital and ICU stay in both groups without a significant difference between them. This large, randomized prospective trial in a cohort of cardiac surgery patients suggests benefit from the routine early, prophylactic administration of vitamin C. Also, the lack of adverse effects, coupled with the minimal expense, supports the notion that this supplementation is a reasonable therapeutic intervention in cardiac surgery patients. Vitamin C supplementation can decrease some postoperative complications and the length of hospital stay in patients undergoing cardiac surgery (14-17)

\section{Authors' Contributions}

Conception: Anita Sadeghpour, Azin Alizadehasl, Majid
Kyavar; Critical review: Anita Sadeghpour, Azin Alizadehasl; Material: all of the authors; Design: Majid Kyavar, Azin Alizadehasl, Tahereh Sadeghi, Behshid Ghadrdoost; Writing: Anita Sadeghpour, Azin Alizadehasl; Edition: all of the authors; Revision: all of the authors.

\section{References}

1. Messaoudi N, De Cocker J, Stockman B, Bossaert LL, Rodrigus IE. Prediction of prolonged length of stay in the intensive care unit after cardiac surgery: the need for a multi-institutional risk scoring system. J Card Surg. 2009;24(2):127-33.

2. Weintraub WS, Jones EL, Craver J, Guyton R, Cohen C. Determinants of prolonged length of hospital stay after coronary bypass surgery. Circulation.1989;80(2):276-84.

3. Giakoumidakis K, Baltopoulos GI, Charitos C, Patelarou E, Galanis P, Brokalaki H. Risk factors for prolonged stay in cardiac surgery intensive care units. Nurs Crit Care. 2011;16(5):243-51.

4. Eslami M, Badkoubeh RS, Mousavi M, Radmehr H, Salehi M, Tavakoli $\mathrm{N}$, et al. Oral ascorbic acid in combination with beta-blockers is more effective than beta-blockers alone in the prevention of atrial fibrillation after coronary artery bypass grafting. Tex Heart Inst J. 2007;34(3):268-74.

5. Nathens AB, Neff MJ, Jurkovich GJ, Klotz P, Farver K, Ruzinski JT, et al. Randomized, prospective trial of antioxidant supplementation in critically ill surgical patients. Ann Surg. 2002;236(6):814-22.

6. DeForge LE, Preston AM, Takeuchi E, Kenney J, Boxer LA, Remick DG. Regulation of interleukin 8 gene expression by oxidant stress. J Biol Chem. 1993;268(34):25568-76.

7. Lo SK, Janakidevi K, Lai L, Malik AB. Hydrogen peroxide-induced increase in endothelial adhesiveness is dependent on ICAM-1 activation. Am J Physiol. 1993;264(4 Pt 1):L406-12.

8. Kanazi GE, El-Khatib MF, Yazbeck-Karam VG, Hanna JE, Masri B, Aouad MT. Effect of vitamin C on morphine use after laparoscopic cholecystectomy: a randomized controlled trial. Can J Anaesth. 2012;59(6):538-43.

9. Rodrigo R, Korantzopoulos P, Cereceda M, Asenjo R, Zamorano J, Villalabeitia E, et al. A randomized controlled trial to prevent post-operative atrial fibrillation by antioxidant reinforcement. $J$ Am Coll Cardiol. 2013;62(16):1457-65.

10. Dehghani MR, Madjidi N, Rahmani A, Asgari B, Rezaei Y. Effect of oral vitamin $C$ on atrial fibrillation development after isolated coronary artery bypass grafting surgery: A prospective randomized clinical trial. Cardiol J. 2014;21(5):492-9.

11. Carnes CA, Chung MK, Nakayama T, Nakayama H, Baliga RS, Piao S, et al. Ascorbate attenuates atrial pacing-induced peroxynitrite formation and electrical remodeling and decreases the incidence of postoperative atrial fibrillation. Circ Res. 2001;89(6):E32-8.

12. Tuman KJ, McCarthy RJ, March RJ, Najafi H, Ivankovich AD. Morbidity and duration of ICU stay after cardiac surgery. A model for preoperative risk assessment. Chest.1992;102(1):36-44.

13. Jian L, Sheng S, Min Y, Zhongxiang Y. Risk factors for endotracheal re-intubation following coronary artery bypass grafting. J Cardiothorac Surg. 2013;8:208.

14. Hein OV, Birnbaum J, Wernecke K, England M, Konertz W, Spies C. Prolonged intensive care unit stay in cardiac surgery: risk factors and long-term-survival. Ann Thorac Surg. 2006;81(3):880-5.

15. El-Chami MF, Kilgo P, Thourani V, Lattouf OM, Delurgio DB, Guyton RA, et al. New-onset atrial fibrillation predicts long-term mortality after coronary artery bypass graft. J Am Coll Cardiol. 2010;55(13):1370-6.

16. Saxena A, Dinh DT, Smith JA, Shardey GC, Reid CM, Newcomb AE. Usefulness of postoperative atrial fibrillation as an independent predictor for worse early and late outcomes after isolated coronary artery bypass grafting (multicenter Australian study of 19,497 patients). Am J Cardiol. 2012;109(2):219-25.

17. Girerd N, Magne J, Pibarot P, Voisine P, Dagenais F, Mathieu P. Postoperative atrial fibrillation predicts long-term survival after aortic-valve surgery but not after mitral-valve surgery: a retrospective study. BMJ Open. 2011;1(2):e000385. 